\title{
Erratum to: HEVC early termination methods for optimal CU decision utilizing encoding residual information
}

\author{
Ting-Lan Lin ${ }^{1} \cdot$ Chi-Chan Chou ${ }^{1}$ Zhaoyi Liu ${ }^{2} \cdot$ Kun-Hsien Tung ${ }^{1}$
}

Published online: 27 July 2016

(C) Springer-Verlag Berlin Heidelberg 2016

\section{Erratum to: J Real-Time Image Proc DOI 10.1007/s11554-016-0608-9}

Unfortunately, the author's photographs were missed in the original publication of the article.

The original article has been updated accordingly. The author's photographs are given below:

Ting-Lan Lin

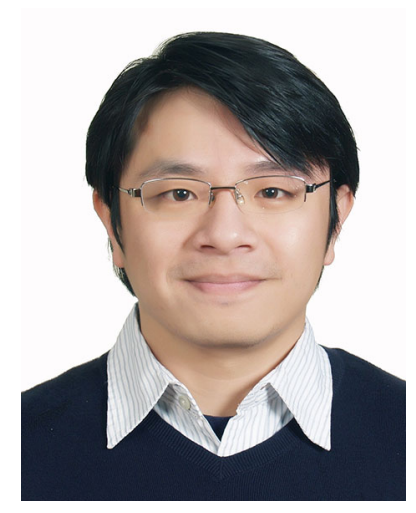

Chi-Chan Chou

The online version of the original article can be found under doi:10.1007/s11554-016-0608-9.

Ting-Lan Lin

tinglan@cycu.edu.tw

Chi-Chan Chou

g10276011@cycu.edu.tw

Zhaoyi Liu

20091305@bit.edu.cn

Kun-Hsien Tung

g10476042@cycu.edu.tw

1 Department of Electronic Engineering, Chung Yuan Christian University, No. 200, Zhongbei Rd., Zhongli City 320, Taoyuan County, Taiwan, ROC

2 School of Information and Electronics, Beijing Institute of Technology, No. 5 Zhongguancunnan Street, Haidian District, Beijing 100081, People's Republic of China 
Zhaoyi Liu

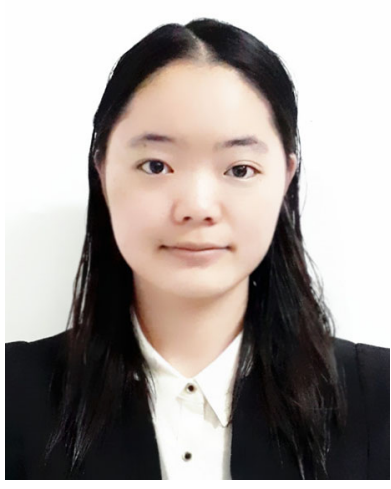

Kun-Hsien Tung

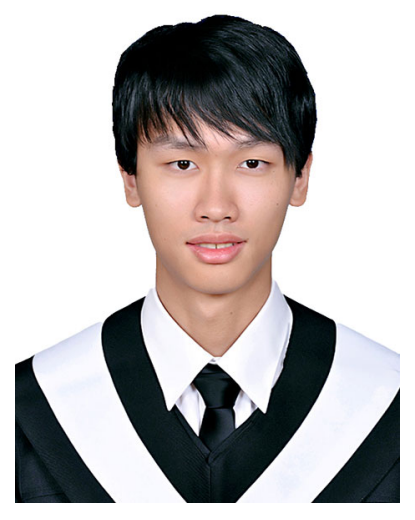

